\title{
Editorial
}

\section{Differential Equations with Applications to Industry}

\author{
Ebrahim Momoniat, ${ }^{1}$ T. G. Myers, ${ }^{2}$ \\ Mapundi Banda, ${ }^{3}$ and Jean Charpin ${ }^{4}$ \\ ${ }^{1}$ Centre for Differential Equations, Continuum Mechanics and Applications, \\ School of Computational and Applied Mathematics, University of the Witwatersrand, \\ Johannesburg, Private Bag 3, Wits 2050, South Africa \\ ${ }^{2}$ Centre de Recerca de Matemàtica, Edifici C, Campus de Bellaterra, Bellaterra, 08193 Barcelona, Spain \\ ${ }^{3}$ Applied Mathematics Division, Department of Mathematical Sciences, Stellenbosch University, \\ Private Bag X1, Matieland 7206, South Africa \\ ${ }^{4}$ MACSI, Department of Mathematics \& Statistics, College of Science \& Engineering, \\ University of Limerick, Limerick, Ireland
}

Correspondence should be addressed to Ebrahim Momoniat, ebrahim.momoniat@wits.ac.za

Received 11 December 2012; Accepted 11 December 2012

Copyright $\odot 2012$ Ebrahim Momoniat et al. This is an open access article distributed under the Creative Commons Attribution License, which permits unrestricted use, distribution, and reproduction in any medium, provided the original work is properly cited.

This special issue is focused on the application of differential equations to industrial mathematics. Of particular interest is the role played by industrial mathematics in the development of new ideas and applications. We are particularly interested in industrial mathematics problems that come from industrial mathematics study group meetings, which take place regularly at universities across the world. These study group meetings are motivated by solving real-world problems that are posed by industry representatives at the start of the meeting. Graduate students and academics then spend one week developing mathematical models that simulate the problems presented. These mathematical models are then solved (usually after some simplification), and conclusions relevant to the real-world problem are made.

This special issue contains a paper that is based on a problem presented by the coal mining industry in South Africa at an industrial mathematics study group meeting. In the paper, the author considers the possible collapse of the roof between the pillar to be mined next in secondary coal mining and the first line of pillar remnants called snooks. Here, the Euler-Bernoulli beam equation is used to model the roof rock between the pillars, which is the working face between two pillars. The model predicts that the beam will break at the clamped end at the pillar. The failure of the beam for different values of the physical parameters is investigated computationally.

Many industrial mathematics problems contain an aspect of heat conduction. This special issue contains a paper in which a new error measure is proposed for the heat balance 
integral method that combines a least-square error with a boundary immobilisation method. The authors show how an optimal heat balance formulation can be obtained by applying their error measure to three basic thermal problems. This new error measure combined with the heat integral method is then applied to two industrially important phase change problems.

Elastic rods are used in many industrial and engineering applications. This special issue also contains another paper that develops a numerical method for special Cosserat rods developed for hyperelastic materials and potential forces. The numerical method preserves the orthonormality of the directors and the conservation of energy of the system of partial differential equations modeling the elastic rods.

In an application to biological modeling, an article developing a mathematical model for collapsing bladder flow is presented in this special issue. The authors derive a coupled system of nonlinear equations derived from the Navier-Stokes equations modeling urinary velocities that depend on the shape of the bladder. Both computational work and specialconfiguration analysis are applied over a range of configurations including results for the circle and sphere as basic cases. The authors also include models of more realistic bladder shapes as well as the end stage of the micturition process where the bladder is relatively squashed down near the urethral sphincter.

The investigation of industrial mathematics problems sometimes leads to the development of new methods of solution of differential equations. This special issue contains a paper on the fractional variational iteration method to determine approximate analytical solutions of nonlinear fractional differential equations. The fractional variational iteration method is applied to the nonlinear fractional Riccati equation with a modified RiemannLiouville derivative. The fractional variational iteration method is shown to be an efficient method for the solution of nonlinear fractional differential equations.

Modeling in industrial mathematics problems with parabolic equations is very common. This special issue also contains a survey paper in which the author investigates the blow-up phenomena for Fujita-type parabolic equations. The author then goes on to discuss various results on the life span of positive solutions for several superlinear parabolic problems.

This special issue has covered both the theoretical and applied aspects of industrial mathematics. Papers contain the development of new mathematical models or well-known models applied to new physical situations as well as the development of new mathematical techniques. It is this multidisciplinary nature of industrial mathematics that makes it a challenging, fruitful, and exciting area of research.

\section{Acknowledgment}

We are grateful to all the authors who have made a contribution to this special issue.

Ebrahim Momoniat

T. G. Myers

Mapundi Banda

Jean Charpin 


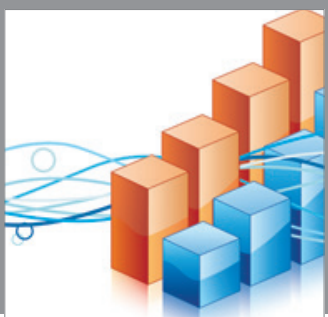

Advances in

Operations Research

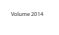

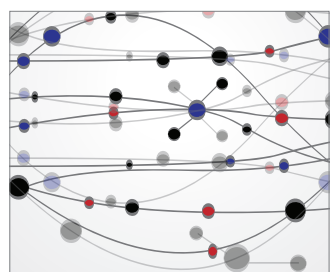

\section{The Scientific} World Journal
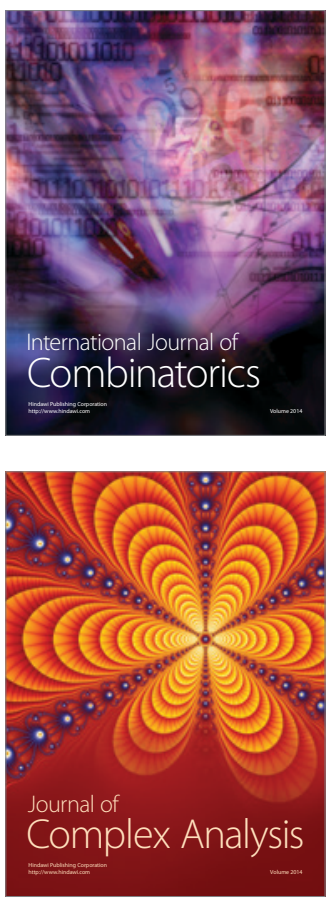

International Journal of

Mathematics and

Mathematical

Sciences
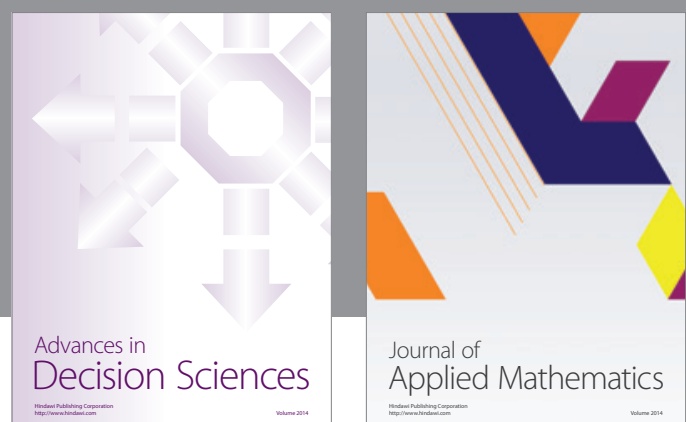

Journal of

Applied Mathematics
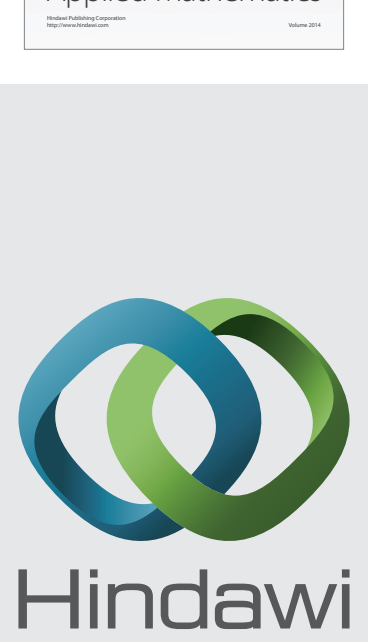

Submit your manuscripts at http://www.hindawi.com
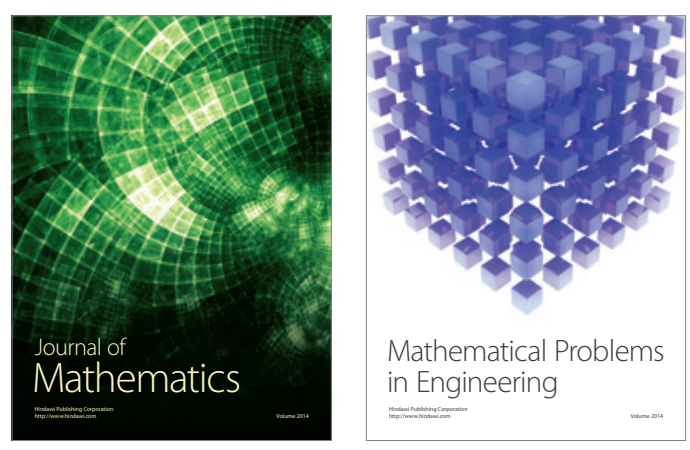

Mathematical Problems in Engineering
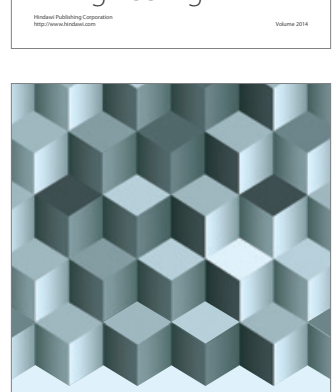

Journal of

Function Spaces
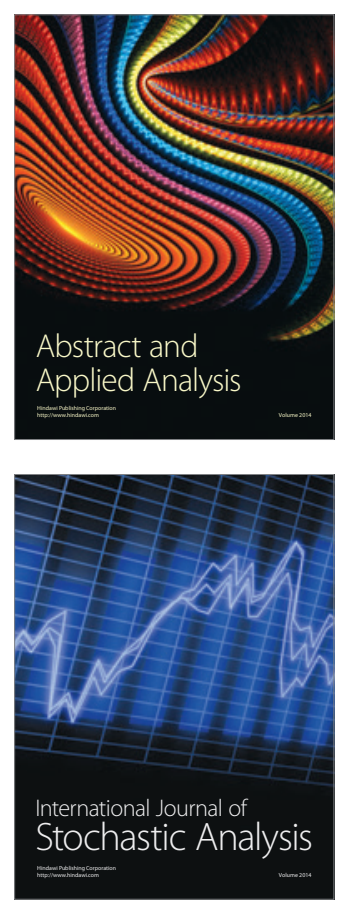

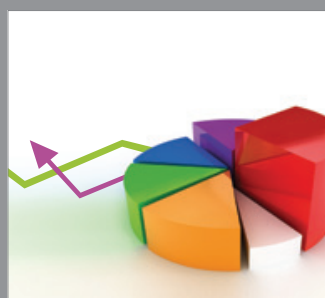

ournal of

Probability and Statistics

Promensencen
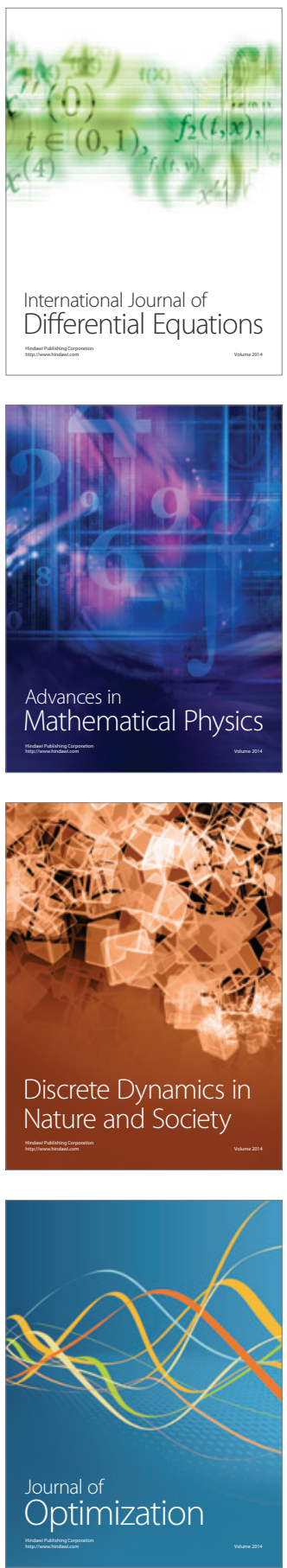